\title{
SMILE CORRECTION IN THE ENMAP GROUND SEGMENT PROCESSOR: A QUALITATIVE ANALYSIS
}

\author{
Maximilian Langheinrich*, Rudolf Richter, Raquel de los Reyes, Gintautas Palubinskas, Tobias Storch \\ DLR, Remote Sensing Technology Institute, Department Photogrammetry and Image Analysis \\ Münchner Str. 20, 82234 Weßling \\ Germany \\ * corresponding author: maximilian.langheinrich@dlr.de
}

\author{
Commission I, WG I/1
}

KEY WORDS: EnMAP, smile correction, smile aware atmospheric correction, inverse atmospheric correction, processing chain

\begin{abstract}
:
The Environmental Mapping and Analysis Program (EnMAP) is an upcoming German hyperspectral satellite mission aiming to observe and characterize the Earth's environment on a global scale. In course of a the development of the EnMAP ground segment processing chain, measures were taken to deal with probable spectral and spatial distortion effects of the sensor, that can only be addressed by post-processing once the satellite platform is launched into orbit. One of these effects is a pixel- and band-wise spectral distortion, the so called sensor smile effect. This paper describes the methods and performance of the smile correction process as implemented for the EnMAP ground segment processing chain, explaining design choices and difficulties currently encountered with the proposed approach and evaluating the results of the smile correction on basis of simulated EnMAP hyperspectral data.
\end{abstract}

\section{INTRODUCTION}

\subsection{The EnMAP hyperspectral imager satellite mission}

The Environmental Mapping and Analysis Program (EnMAP) is an upcoming German hyperspectral satellite mission aiming to observe and characterize the Earth's environment on a global scale.

It will cover the spectral range from $429 \mathrm{~nm}$ to $2431 \mathrm{~nm}$ with a spectral sampling distance varying between $4.8 \mathrm{~nm}$ and $12.0 \mathrm{~nm}$. It will acquire $30 \mathrm{~km}$ in the across-track direction with a ground sampling distance of $30 \mathrm{~m}$ and the across-track tilt capability of $30^{\circ}$ will enable a target revisit time of less than 4 days. The launch is scheduled for 2020 with an operational lifetime of 5 years. The mission is in its production phase. The hyperspectral image products at different processing levels will be freely available to the scientific user community for measuring and analyzing bio-, geochemical, and physical parameters characterizing the Earth's surface. This encompasses agriculture, forestry, soil and geological environments, as well as coastal zones and inland waters [Storch et al., 2018].

\subsection{Smile correction in EnMAP processing}

During the launch of a satellite payload strong forces affect the sensitive instrumentation which may cause small mechanical deformations that result in a sensor behaviour deviating from the characteristics determined in on-ground calibration. One effect that may occur in terms of a spectral misalignment (which is not necessarily only caused during the launch) of the measured wavelength is the so called spectral smile. This error, which can be specified as a change of dispersion angle with field position [Fisher et al, 1998], cannot be corrected in terms of sensor adjustment when the satellite platform is in orbit. Although it is expected, that in the case of an occurring smile error after launch, the spectral accuracy concerning this deviation will not violate the 0.2 pixel requirement for the EnMAP Mission, the ground segment processing chain is prepared to handle a potential smile effect of a greater magnitude.

The proposed approach of smile correction in the EnMAP ground segment processor results from decisions concerning the design of the EnMAP mission's sensor payload Hyper Spectral Imager (HSI). The instrument's overall spectral range from 429 $\mathrm{nm}$ to $2431 \mathrm{~nm}$ is covered by two individual spectrometers, with the VNIR ranging from the visible spectrum to the very-near infrared and the SWIR covering the short-wave infrared. This construction choice leads to two problems concerning the nominal smile correction solution as part of the DLR atmospheric correction (AC) framework PACO [De los Reyes et al., 2018].

First smile correction is nominally applied to bottom of atmosphere (BOA) reflectances. In order to achieve the highest possible atmospheric correction accuracy, both hyperspectral cubes have to be merged to one cube to cover the whole spectral range of the sensors. A smile-aware atmospheric correction is applied to the top of atmosphere (TOA) radiances taking the shifted wavelengths of the individual bands into account. The look-up-tables used in the nominal atmospheric correction approach are interpolated to the shifted wavelength on the fly. After retrieving the BOA reflectances a linear interpolation in spectral dimension, the actual smile correction is applied to shift the values to the according nominal central wavelength of the band. This process has by implementation to be applied in the L1B processor of the processing chain to enable a column-wise atmospheric correction. Doing so increases the processing performance as all column pixels of one band can be corrected simultaneously. For EnMAP the VNIR and SWIR cubes are not spatially aligned in sensor geometry, featuring a spatial shift of 
approximately 20 scanlines in flight direction of the satellite. As the highly accurate spatial matching of the two hyperspectral cubes is conducted during the orthorectification process in processing level L1C resulting in a geocoded merged cube the smile correction has to be applied on the individual cubes in level L1B. Smile-aware atmospheric correction must therefore be inherently applied to the individual cubes. This decreases the overall quality of the atmospheric correction result, especially in the SWIR where the lack of channels in the visible spectral range disables the ability to classify individual earth surface features on a pixel wise basis, especially in terms of dense dark vegetation pixels, which are needed to retrieve important atmospheric parameters e.g. aerosol optical thickness.

These issues and the given condition that user products of the subsequent processing level $\mathrm{L} 1 \mathrm{C}$ are required to be delivered in TOA radiances, create the necessity that the smile-corrected BOA reflectances are calculated back to smile-corrected at.sensor measurements. It is required to invert the atmospheric correction model as part of the intermediate L1B processing step.

The resulting radiances then are used as an input to the L1C processor resulting in smile-corrected, orthorectified and merged images cubes featuring TOA radiance values. These smile free images can then be processed by the L2A processor performing the nominal, highly accurate atmospheric correction of the PACO environment. In order to enable the correct inversion of the AC, a simplified model has to be assumed as explained in 2.1 .

This paper aims to deliver an in-depth description of the smileaware atmospheric correction process, the interpolation of the reflectance values to the nominal centre wavelength and the inversion of the atmospheric correction model (BOA to TOA) in level L1B. Results are analysed on a qualitative level, showing the feasibility of the proposed approach.

\section{METHODOLOGY}

\subsection{Smile-aware atmospheric correction}

As stated above, in order to enable atmospheric correction of the cubes produced by the individual EnMAP sensors, a simplified approach must be applied, as none of the separate cubes features the full range of spectral bands necessary for nominal (L2A) atmospheric correction processing. A simplified approach with regard to smile correction is characterized by the following atmospheric correction settings:

Constant Visibility - $23 \mathrm{~km}$, no bidirectional reflectance distribution function (BRDF) correction, no spectral interpolation of absorption bands, clouds are not masked, no adjacency correction, no clipping of negative values. Additionally a accurate pixel-wise variable water vapor map has to be retrieved to correctly account for the shifted wavelengths caused by the sensor smile, particularly in the absorption regions of the spectrum. Therefore a DEM in sensor geometry is needed for the process. These assumptions render the atmospheric correction process invertible.

The smile effect as it is apparent in the simulated EnMAP data used during the development of the processor can be seen in

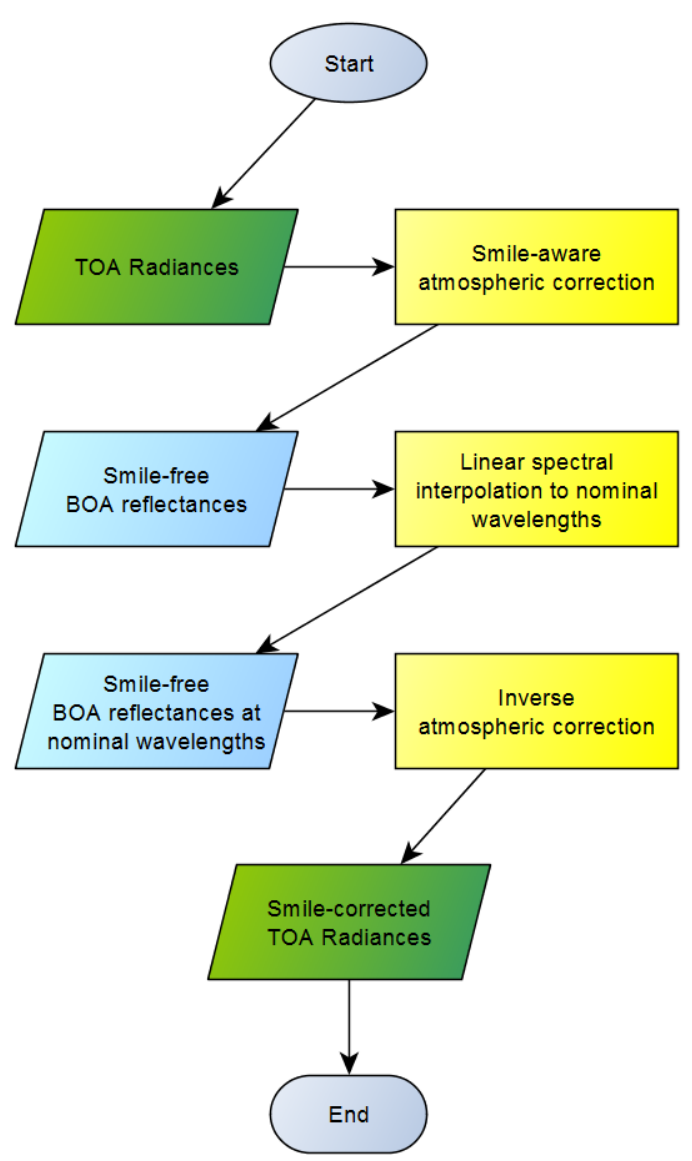

Figure 1. Flowchart of EnMAP smile correction processing.

Figure 1 and is described for the atmospheric correction approach using spectral smile look-up tables (LUTs) in terms of a per-band fourth order polynomial of wavelengths shifts relative to the nominal detector band wavelength:

$$
P_{n}(j)=\sum_{i=0}^{4} a_{n, i} \cdot j_{n}^{i}
$$

where $a_{i}$ are the polynomial coefficients, $j$ is the across-track pixel number (ranging from 0 to 999 for EnMAP) and $n$ is the respective detector band. The atmospheric correction approach using LUTs and considering smile on a column-wise basis are described in detail in [Richter et al, 1990] and [Richter et al, 2011] as follows:

1. High spectral resolution $(0.6 \mathrm{~nm})$ atmospheric LUTs containing the radiative transfer functions for a discrete 6D parameter space are loaded.

2. The LUTs are interpolated for scene related parameters like i.e. solar and sensor geometry, ground elevation.

3. The interpolated database is convolved with the spectral response function of each band of the sensor. The actual response function is the sum of the nominal response function for the central wavelength of the band and the local sensor shift described by the polynomial. 
4. Smile-aware water vapor retrieval on a column-wise basis.

5. Smile-aware, simplified surface reflectance retrieval on a column-wise basis.

This process results in intermediate BOA surface reflectances according to the individual wavelength of each particular pixel of a band. A representative reflectance spectrum of a vegetation pixel can be seen in Figure 2.

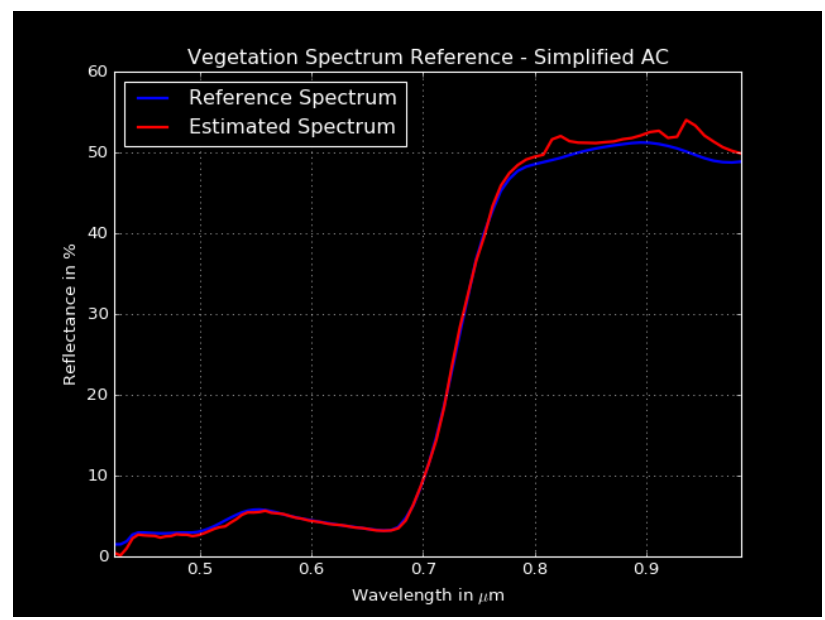

Figure 2. Exemplary intermediate BOA reflectance spectrum of VNIR cube.

\subsection{Smile correction (spectral linear interpolation of bands)}

In the next step linear interpolation in the spectral dimension of the particular hyperspectral cube is applied on a pixel-wise basis. This shifts the resulting BOA reflectances to the nominal centre wavelength, as defined by pre-launch detector calibration, of each band.

\subsection{Inverse AC model}

\subsubsection{Smile-free inverse AC}

In order to be able to deliver TOA radiances with the L1C EnMAP user product and to apply the nominal atmospheric correction in the L2A processor, the inverse AC model has to be applied to the smile corrected reflectances. Preliminary, to evaluate the accuracy of the inverse model of the simplified atmospheric correction process, simplified AC was applied to the smile free TOA radiance input VNIR and SWIR cubes and the reverse model was calculated from the reflectance values acquired in the preceding step according to the inverse of the model equation given in [Richter, 1990]:

$$
L_{T O A}^{i}=\frac{\left[\frac{\left(\rho_{k}^{i} \cdot a_{1}+a_{0}\right) \cdot E_{s}\left(\lambda_{i}\right) \cos \theta_{s}}{\pi d^{2}}\right]-c_{0}^{i}}{c_{1}^{i}}
$$

where $\rho_{k}^{i}$ is the BOA reflectance value of pixel $\mathrm{k}$ in band $\mathrm{i}$, $E_{S}\left(\lambda_{i}\right)$ the extraterrestrial solar irradiance at nominal wavelength $\lambda$ for band i, $\theta_{s}$ the solar zenith angle, $d$ the EarthSun distance in astronomical units, $c_{0}^{i}$ and $c_{1}^{i}$ the quantization offset and gain coefficient of the radiance DN ( 0 and 1 in
EnMAP internal processing) and $a_{0}$ and $a_{1}$ the coefficients of the model-derived planetary albedo.

As all parameters of the model are known from step 2.1 and kept for inversion purpose, it can be postulated that the absolute error between input TOA radiances and radiances resulting from the reverse processing is zero for the smile free situation. This was tested on nominal, smile free EnMAP cubes, resulting in identical TOA radiance images before and after the process, proofing that the inverse $\mathrm{AC}$ calculation can therefore be judged as valid, as it does not introduce additional errors.

\subsubsection{Smile-corrected inverse AC}

For the inverse atmospheric correction on the smile corrected reflectance value, atmospheric look-up tables have to be interpolated on basis of the nominal response functions of the detector and the parameters of the atmospheric correction model have to be recalculated using the nominal, interpolated LUTs.

The same inverse model as described in 2.3.1 is used to retrieve smile corrected TOA radiances for $\mathrm{L} 1 \mathrm{C}$ user product and $\mathrm{L} 2 \mathrm{~A}$ processing input. Figure 5 shows the relative errors for the inversion results from smile corrected reflectance values.

\section{DATA}

The data used in this evaluation is a simulated EnMAP datatake with the size of 1000 by 3072 pixels which corresponds to a surface cover area of $30 \mathrm{~km}$ by $92.16 \mathrm{~km}$. Two hyperspectral image cubes for the VNIR and SWIR spectral region of the HSI instrument were used, featuring a spatial shift in y-dimension of approximately 19 pixels. The VNIR dataset features 88 spectral channels covering the spectral range of $429 \mathrm{~nm}$ to $985 \mathrm{~nm}$. The SWIR dataset features 154 spectral channels ranging from 904 $\mathrm{nm}$ to $2431 \mathrm{~nm}$. The true color image of the simulated VNIR scene can be seen in Figure 4.

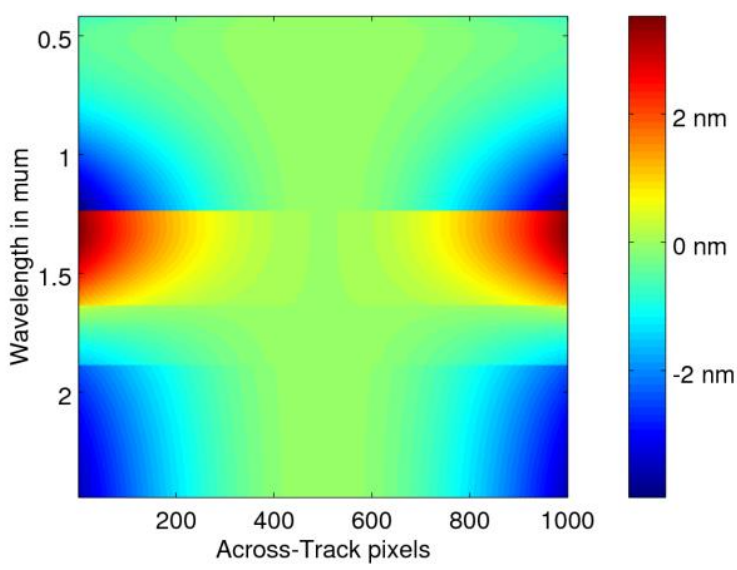

Figure 3. Smile effect for simulated EnMAP test scene. Colorbar showing the magnitude of spectal smile shift per pixel.

TOA radiances of the described area were simulated from Sentinel-2 BOA reflectances using an inverse atmospheric correction model with a radiative transfer model corresponding to MODTRAN 4, New_kuruc1997 sun model, a MODTRAN resolution of $1 \mathrm{~cm}^{-1}$, monochromatic LUTS with a resampling distance of $1 \mathrm{~nm}$ and the MODTRAN 4 water vapor absorption 
model. No digital elevation model was used in the simulation. Instead the average height of the scene of $1529 \mathrm{~m}$ was used as a flat plane approximation. Spectral smile was simulated as depicted in Figure 3.

\section{EXPERIMENTS}

The described datasets were used as inputs for the current incarnation of the EnMAP development processor for Level 1B. The resulting smile corrected TOA radiances were compared with available smile free datasets of the used scene.

\section{RESULTS}

Concerning the backward calculation of smile corrected BOA radiances, errors are introduced in the inverse $\mathrm{AC}$ model as can be seen in Figure 5.

The error sources can be backtracked to two particular sources. The large errors of approx. $100 \%$ relative error in the SWIR cube TOA dataset (at $\sim 1.4$ and $\sim 1.85 \mu \mathrm{m}$ in Figure 5) are caused by the missing interpolation of water vapor absorption bands during the simplified atmospheric correction. As the signal contained in the affected input bands is close to zero, the estimation of surface leads to highly inaccurate and numerically very large BOA reflectance values. As this intermediate reflectance acts as an input for the inverse AC process, the significantly wrong values (at $\sim 1.4$ and $\sim 1.85 \mu \mathrm{m}$ in Figure 5) propagate to the newly calculated TOA radiances. This error can and is addressed by the interpolation of the particular absorption bands during the full atmospheric correction in the L2A processor. As for this the mean error given in Figure 5 was calculated without taking the error-prone absorption bands into account.

Further smaller errors (as observed over the whole spectral range in the VNIR plot of Figure 5) are caused by the fact that for the smile case the AC model used to apply the smile correction cannot be used in its inverted form in practice. If this was the case, the smile effect would be reapplied to the BOA reflectance. Therefore an inversion of the AC model without implicit smile correction must be used. This introduces uncertainties to the corresponding TOA radiances. Unfortunately this problem cannot be avoided at the moment.

\section{CONCLUSION}

The experiments conducted as an evaluation of the proposed smile correction method for EnMAP satellite data show that the overall process is feasible for the handling of a smile effect in the image data. The algorithm is able to produce smile free boa reflectance with a mean relative TOA radiance error of $-0.30 \%$ and $0.61 \%$ for VNIR and SWIR respectively. These values can be considered as accurate within the requirements of the EnMAP ground processing chain.

\section{ACKNOWLEDGEMENTS}

Supported by the DLR Space Administration with funds of the German Federal Ministry of Economic Affairs and Technology on the basis of a decision by the German Bundestag (50 EE 0850).

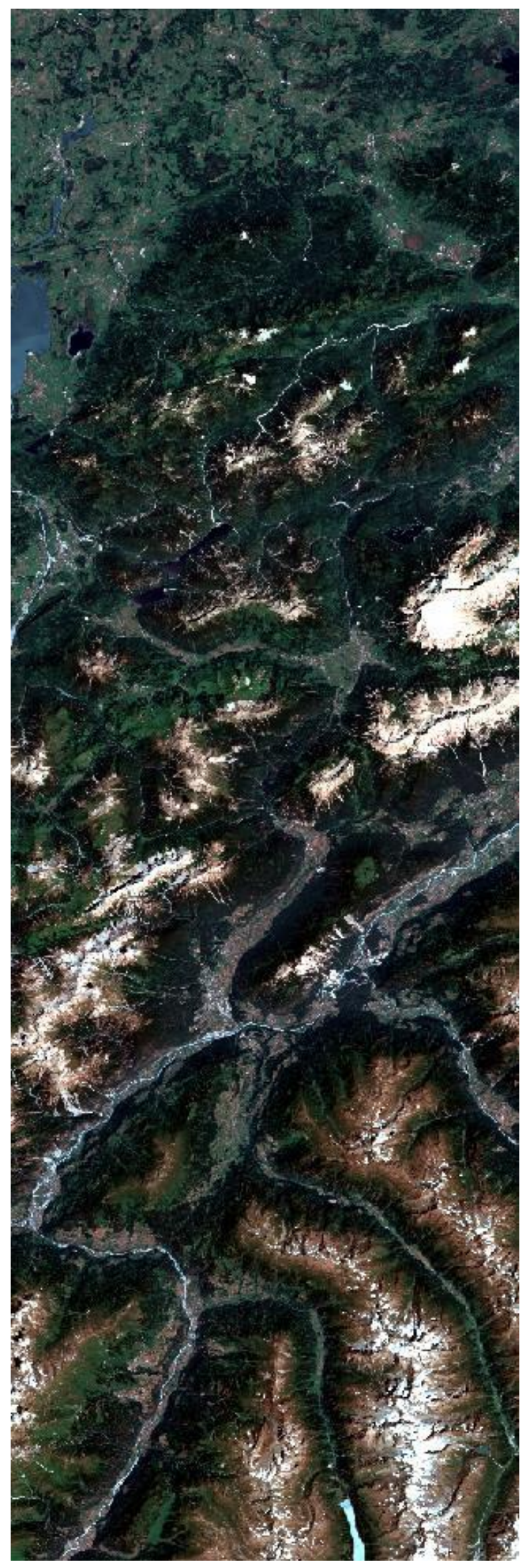

Figure 4. Truecolor scene of simulated EnMAP VNIR cube. 


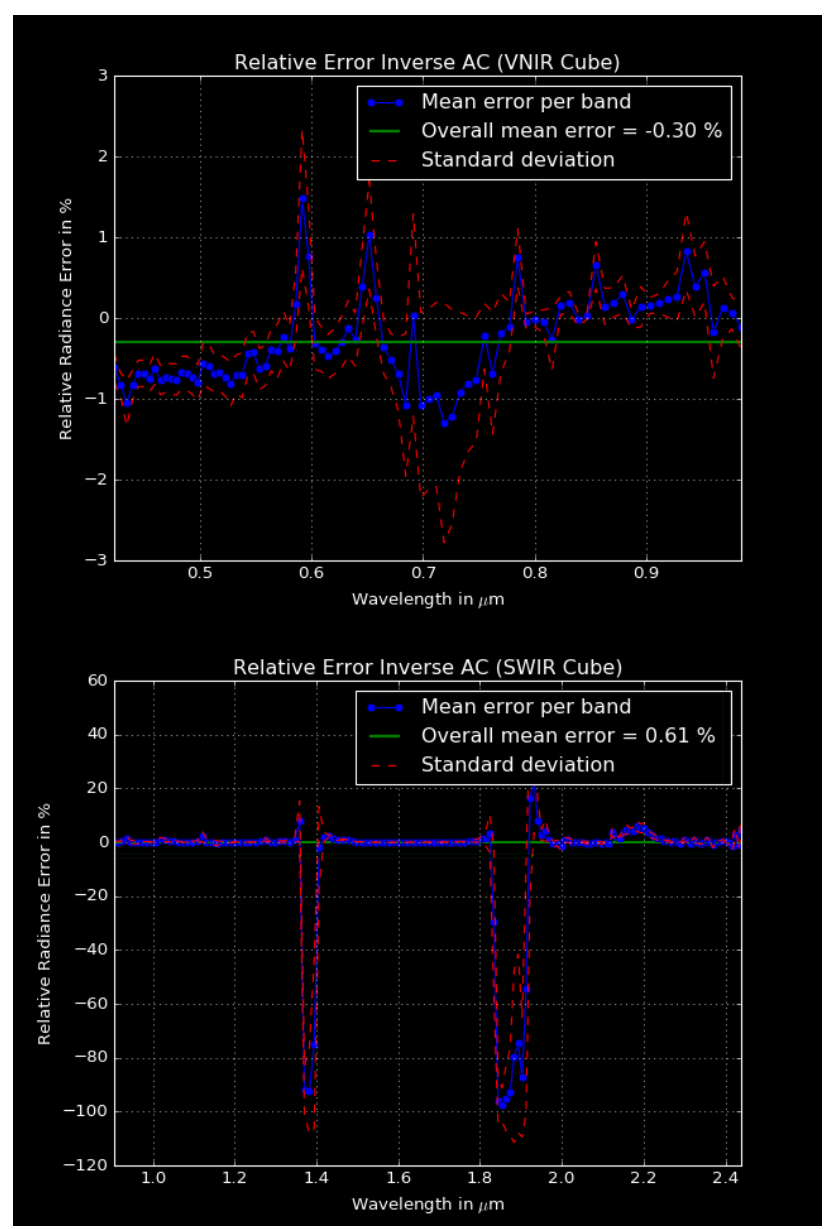

Figure 5. Relative error of VNIR (top) and SWIR (bottom) between smile corrected TOA radiances and smile free TOA radiances of the simulated scene.

\section{REFERENCES}

De los Reyes, R., Richter, R., Langheinrich, M., Pflug, B., \& Schwind, P. (2018). Validation of a new atmospheric correction software using AERONET reference data PACO: Python-based Atmospheric COrrection. Poster session LPVE 2018, Frascati, Italy.

Fisher, J., Baumback, M. M., Bowles, J. H., Grossmann, J. M., \& Antoniades, J. A. (1998, October). Comparison of low-cost hyperspectral sensors. In Imaging Spectrometry IV (Vol. 3438, pp. 23-31). International Society for Optics and Photonics.

Richter, R. (1990). A fast atmospheric correction algorithm applied to Landsat TM. International journal of Remote Sensing, 11, 159-166.

Richter, R., Schlapfer, D., \& Muller, A. (2011). Operational atmospheric correction for imaging spectrometers accounting for the smile effect. IEEE Transactions on Geoscience and Remote sensing, 49(5), 1772-1780.

Storch, T., Honold H.P., Guanter' L., Schwind, P., Mücke, M., Segl, K. \& Fischer, S. (2018). The Imaging Spectroscopy Mission EnMAP-ITS Status and Expected Product.WHISPERS 2018, Amsterdam. 